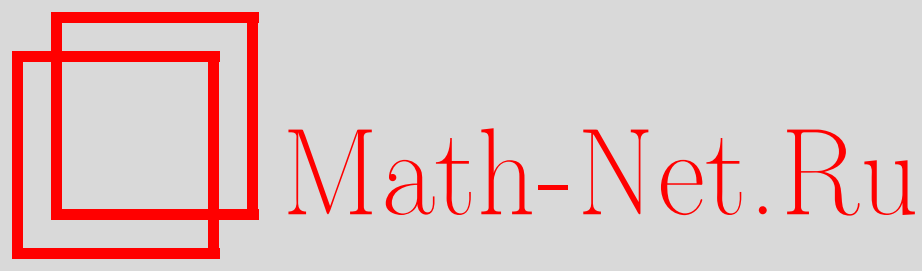

Ю. С. Харин, Е. В. Вечерко, Распознавание вкраплений в двоичную цепь Маркова, Дискрет. матем., 2015, том 27, выпуск 3, 123-144

DOI: https://doi.org/10.4213/dm1339

Использование Общероссийского математического портала Math-Net.Ru подразумевает, что вы прочитали и согласны с пользовательским соглашением http://www . mathnet.ru/rus/agreement

Параметры загрузки:

IP: 54.162 .85 .209

26 апреля 2023 г., 06:31:54

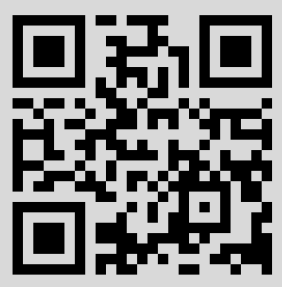




\title{
Распознавание вкраплений в двоичную цепь Маркова
}

\author{
() 2015 г. Ю. С. Харин*, Е. В. Вечерко**
}

Рассматриваются возникающие в стеганографии задачи обнаружения вкраплений и статистического оценивания позиций, в которые вкраплены биты сообщения. В качестве моделей контейнера используются двоичные стационарные цепи Маркова как с известными, так и неизвестными матрицами переходных вероятностей. Построены статистические критерии, позволяющие выявить факт наличия вкраплений, основанные на статистиках серий и статистике отношения правдоподобия. Найдена асимптотическая мощность статистических критериев, основанных на статистиках серий, для семейства контигуальных альтернатив. Разработан алгоритм статистического оценивания позиций, в которые вкраплены биты сообщения, имеющий полиномиальную сложность. Представлены результаты компьютерных экспериментов.

Ключевые слова: стеганография, модель вкраплений, цепь Маркова, статистический критерий, мощность, число серий.

\section{1. Введение}

В статье рассматривается актуальная проблема стеганографической защиты информации - проблема распознавания вкраплений, которая состоит в построении статистических критериев проверки гипотез о наличии вкраплений и статистических оценок позиций (точек) вкраплений.

Задача обнаружения (выявления) факта вкраплений рассматривалась в $[1,2,3,4]$; в этих работах предполагалось, что вероятностная модель контейнера полностью известна. Так, в [1] построены статистические критерии проверки гипотез о наличии вкраплений для случая, когда моделью исходной последовательности является бернуллиевская схема независимых испытаний, и показано, что обнаружить наличие вкраплений невозможно, если доля вкраплений растет по порядку медленнее, чем длина исходной последовательности. Аналогичный факт установлен в [3]. В [2] построен наиболее мощный критерий проверки гипотез о наличии вкраплений для модели на основе бернуллиевской схемы независимых испытаний, построены статистические оценки доли вкраплений. В [5] построены и исследованы статистические

*Место работы: Белорусский государственный университет, e-mail: kharin@bsu.by

${ }^{* *}$ Место работы: Белорусский государственный университет, e-mail: vecherko@bsu.by 
оценки параметров модели вкраплений в двоичную цепь Маркова, вычисление которых позволяет делать начальные выводы о доле вкраплений. Следует отметить, что большинство работ по обнаружению факта вкраплений основано на эмпирических характеристиках последовательностей, в которых для проверки гипотез о наличии вкраплений используются методы дискриминантного анализа. Отметим также, что рассматриваемые задачи распознавания вкраплений близки к задачам выявления отклонений выходных последовательностей криптографических генераторов от равномерно распределенных случайных последовательностей [6].

Целью данной статьи является развитие исследований, начатых в [5], направленное на построение и анализ статистических критериев проверки гипотез о наличии вкраплений, а также на разработку алгоритма статистического оценивания точек вкраплений.

Статья имеет следующую структуру. В разделе 2 дается описание математической $(q, r)$-блочной модели вкраплений в двоичную цепь Маркова. В разделе 3 построены статистические критерии проверки гипотез о наличии вкраплений на основе статистики числа серий и статистики коротких серий, в разделе 4 - на основе статистики отношения правдоподобия. В разделе 5 разработан алгоритм полиномиальной сложности статистического оценивания точек вкрапления. Раздел 6 содержит результаты компьютерных экспериментов.

\section{2. Математическая модель вкраплений}

Определим обобщенную $(q, r)$-блочную модель вкраплений, частный случай которой предложен авторами в [5]. Введем обозначения: $(\Omega, F, \mathbf{P})$ - основное вероятностное пространство, $V=\{0,1\}$ - двоичный алфавит, $V_{T}-$ пространство двоичных $T$-мерных векторов, $\mathcal{O}(\cdot)$ - символ Ландау “о большое", $\mathbb{N}$ - множество натуральных чисел, $I\{A\}$ - индикатор события $A, u_{t_{1}}^{t_{2}}=\left(u_{t_{1}}, \ldots, u_{t_{2}}\right) \in V_{t_{2}-t_{1}+1}\left(t_{1}, t_{2} \in \mathbb{N}, t_{1} \leqslant t_{2}\right)$ - двоичная строка из $t_{2}-t_{1}+1$ последовательных символов некоторой последовательности $\left\{u_{t}: t \in \mathbb{N}\right\}, w(\cdot)$ - вес Хэмминга, $\mathfrak{L}\{\xi\}$ - закон распределения вероятностей случайной величины $\xi, \mathfrak{B}(\theta)$ - закон распределения вероятностей Бернулли с параметром $\theta \in[0,1]: \mathbf{P}\{\xi=1\}=1-\mathbf{P}\{\xi=0\}=\theta, \Phi(\cdot)$ - функция распределения стандартного нормального закона $\mathcal{N}(0,1)$.

Как обосновано в [5], адекватная модель контейнера для встраивания сообщения двоичная последовательность $x_{1}^{T}=\left(x_{1}, x_{2}, \ldots, x_{T}\right) \in V_{T}, x_{t} \in V, t=1, \ldots, T$, длины $T$, являющаяся однородной двоичной цепью Маркова 1-го порядка с симметричной матрицей вероятностей одношаговых переходов $P$ :

$$
P=P(\varepsilon)=\frac{1}{2}\left(\begin{array}{cc}
1+\varepsilon & 1-\varepsilon \\
1-\varepsilon & 1+\varepsilon
\end{array}\right), \mathbf{P}\left\{x_{t} \oplus x_{t+1}\right\}=\frac{1}{2}(1-\varepsilon),|\varepsilon|<1
$$

Здесь $\varepsilon$ - параметр модели: случай $\varepsilon=0$ соответствует схеме независимых испытаний и исследован в [1]; случай $\varepsilon>0$ учитывает зависимость типа притяжения, $\varepsilon<0$ - зависимость типа отталкивания. Отметим, что цепь Маркова (1) удовлетворяет 
условиям эргодичности [7] и имеет равномерное стационарное распределение вероятностей $(1 / 2,1 / 2)$. Далее будем полагать, что цепь Маркова (1) является стационарной, так что ее начальное распределение вероятностей совпадает с равномерным распределением.

В связи с тем, что на практике [5] сообщение перед вкраплением в контейнер подвергается криптографическому преобразованию, далее полагаем, что сообщение $\xi_{1}^{M}=\left(\xi_{1}, \ldots, \xi_{M}\right) \in V_{M}, M \leqslant T$, является последовательностью $M$ независимых бернуллиевских случайных величин:

$$
\mathfrak{L}\left\{\xi_{t}\right\}=\mathfrak{B}\left(\theta_{1}\right), \mathbf{P}\left\{\xi_{t}=j\right\}=\theta_{j}, j \in V, \theta_{1}=1-\theta_{0}, t=1, \ldots, M .
$$

Стегоключ $\gamma_{1}^{T}=\left(\gamma_{1}, \ldots, \gamma_{T}\right) \in V_{T}$, определяет точки (моменты времени), в которых биты сообщения $\xi_{1}^{M}$ вкрапляются в последовательность $x_{1}^{T}$. Введем в рассмотрение специальную $(q, r)$-блочную модель стегоключа $\gamma_{1}^{T}(q, r \in \mathbb{N}, r \leqslant q)$, предполагая, что длина последовательности $x_{1}^{T}$ кратна $q: T=K q$.

Пусть $\zeta_{k} \in V, \mathfrak{L}\left\{\zeta_{k}\right\}=\mathfrak{B}(\delta), k=1, \ldots, K,-$ вспомогательные независимые случайные величины, которые определяют выбор блоков $\left\{x_{(k)}=x_{(k-1) q+1}^{k q}\right\}$ для вкрапления сообщения $\xi_{1}^{M}$ : если $\zeta_{k}=1$, то в $r$ случайно (наудачу) выбранные биты блока $x_{(k)}$ вкрапляются $r$ последовательных бит сообщения; если $\zeta_{k}=0$, то вкрапление в блок $x_{(k)}$ не производится; $G^{(q, r)}=\left\{g_{1}^{(q, r)}, \ldots, g_{C_{q}^{r}}^{(q, r)}\right\}=\left\{u_{1}^{q} \in V_{q}: w\left(u_{1}^{q}\right)=r\right\}-$ множество, состоящее из $C_{q}^{r}$ лексикографически упорядоченных двоичных векторов длины $q$ с весом Хемминга $r ; g_{1}, g_{2}, \ldots$ - независимые случайные величины, $g_{k}$ имеет равномерное распределение вероятностей на множестве $\left\{1, \ldots, C_{q}^{r}\right\}$ :

$$
\mathbf{P}\left\{\gamma_{(k)}=g_{i}^{(q, r)} \mid \zeta_{k}=1\right\}=\mathbf{P}\left\{g_{k}=i\right\}=\frac{1}{C_{q}^{r}}
$$

В $(q, r)$-блочной модели вкраплений последовательность $\gamma_{1}^{T}$ состоит из блоков длины $q: \gamma_{(1)}=\gamma_{1}^{q}, \gamma_{(2)}=\gamma_{q+1}^{2 q}, \ldots, \gamma_{(K)}=\gamma_{(K-1) q+1}^{K q}$,

$$
\gamma_{(k)}=\left\{\begin{array}{ll}
\underbrace{(0, \ldots, 0}_{q}), & \zeta_{k}=0, \\
g_{i}^{(q, r)} \in G^{(q, r)}, & \zeta_{k}=1, g_{k}=i,
\end{array} \quad k=1, \ldots, T / q,\right.
$$

параметр $\delta$ характеризует долю вкраплений. Отметим, что для $(q, r)$-блочной модели вкраплений максимальная пропускная способность стегосистемы равна $\mathrm{Tr} / q$ бит, а мощность множества всевозможных стегоключей

$$
\Gamma^{(q, r)}=\left\{G^{(q, r)} \cup\{(0, \ldots, 0)\}\right\}^{T / q}
$$

равна $\left|\Gamma^{(q, r)}\right|=\left(1+C_{q}^{r}\right)^{T / q}$. В случае $q=r=1$ имеет место классическая модель [5] побитового вкрапления, $\left|\Gamma^{(1,1)}\right|=2^{T}$.

Случайная стегопоследовательность $Y_{1}^{T}=\left(Y_{1}, \ldots, Y_{T}\right)$ для наиболее распространенных в стеганографии методов вкрапления "LSB replacement" и " \pm embedding" [8] порождается последовательностями $\left\{x_{t}\right\},\left\{\xi_{t}\right\},\left\{\gamma_{t}\right\}$ следующим функциональным преобразованием:

$$
Y_{t}=x_{t} \oplus \gamma_{t} x_{t} \oplus \gamma_{t} \xi_{\tau_{t}}= \begin{cases}x_{t}, & \gamma_{t}=0, \\ \xi_{\tau_{t}}, & \gamma_{t}=1\end{cases}
$$


где $\tau_{t}=\sum_{j=1}^{t} \gamma_{j}$. Последовательности $\left\{x_{t}\right\},\left\{\xi_{t}\right\},\left\{\gamma_{t}\right\}$ предполагаются независимыми в совокупности.

Отметим, что при $r=1$ представленная здесь модель превращается в $q$-блочную модель, рассмотренную в [5].

С практической точки зрения наибольшего внимания в рамках рассматриваемой здесь марковской модели вкраплений (1)-(4) заслуживает наиболее трудный для обнаружения вкраплений случай, когда $\theta_{0}=\theta_{1}=1 / 2$ в $(2)$, так как в этом случае при вкраплении (4) одномерное распределение вероятностей не искажается:

$$
\mathbf{P}\left\{Y_{t}=1\right\}=\mathbf{P}\left\{Y_{t}=0\right\}=\mathbf{P}\left\{x_{t}=1\right\}=\mathbf{P}\left\{x_{t}=0\right\}=1 / 2, \quad t=1,2, \ldots, T .
$$

Еще одним обоснованием актуальности этого случая, который и рассматривается далее в статье, является использование на практике предварительного криптографического преобразования сообщения, устраняющего неравномерность вероятностного распределения символов.

\section{3. Обнаружение вкраплений на основе статистик се- рий}

3.1. Использование статистики числа серий. Определим две гипотезы о значении доли вкраплений $\delta \in[0,1]$ :

$$
H_{0}:\{\delta=0\}, H_{1}:\{\delta>0\} .
$$

Гипотеза $H_{0}$ означает, что вкрапления отсутствуют и стегопоследовательность $Y_{1}^{T}$ совпадает с контейнером $x_{1}^{T}$. Сложная альтернатива $H_{1}$ означает наличие вкраплений некоторой неизвестной доли $\delta>0$. Если параметр контейнера $\varepsilon$ известен, то нулевая гипотеза, которую будем обозначать $H_{0, \varepsilon}$, - простая, в противном случае $H_{0}$ - также сложная гипотеза. Если справедлива гипотеза $H_{0}$, то вероятностную меру $\mathbf{P}$ будем обозначать $\mathbf{P}_{0}$, в противном случае $-\mathbf{P}_{\delta}$; аналогично обозначаются моменты случайных величин. Распределения $\mathbf{P}_{0}, \mathbf{P}_{\delta}$ найдены в [5].

Лемма 1. Распределение вероятностей стегопоследовательности $Y_{1}^{T}$ при выполнении гипотезы $H_{0, \varepsilon}$ имеет вид

$$
\mathbf{P}_{0}\left\{Y_{1}^{T}=y_{1}^{T}\right\}=\mathbf{P}_{0}\left\{x_{1}^{T}=y_{1}^{T}\right\}=2^{-T}(1-\varepsilon)^{B_{T}-1}(1+\varepsilon)^{T-B_{T}},
$$

где

$$
B_{T}=B_{T}\left(y_{1}^{T}\right)=1+\sum_{t=1}^{T-1} y_{t} \oplus y_{t+1}
$$

- минимальная достаточная статистика при $H_{0, \varepsilon}$.

Статистика $B_{T}$ есть так называемая "статистика знакоперемен" [9], число серий. В силу (1) при выполнении гипотезы $H_{0}$ последовательность индикаторов $I\left\{Y_{t} \oplus Y_{t+1}=1\right\}$ смены состояний состоит из независимых случайных величин с распределением Бернулли $\mathfrak{B}\left(2^{-1}(1-\varepsilon)\right)$. Используя точное биномиальное распределение вероятностей статистики $B_{T}$ при известном значении $\varepsilon$ по заданной вероятности ошибки первого рода $\alpha$ можно построить рандомизированный статистический 
критерий проверки гипотез о наличии вкраплений, однако на практике удобнее использовать его асимптотический вариант при $T \rightarrow \infty$, задаваемый критической областью

$$
\begin{array}{ll}
\mathscr{X}_{1 \alpha}^{B+}=\left\{y_{1}^{T}: B_{T} \geqslant 1+\frac{1}{2} T(1-\varepsilon)-\frac{1}{2} t_{\alpha} \sqrt{T\left(1-\varepsilon^{2}\right)}\right\} & \text { при } \varepsilon>0, \\
\mathscr{X}_{1 \alpha}^{B-}=\left\{y_{1}^{T}: B_{T} \leqslant 1+\frac{1}{2} T(1-\varepsilon)+\frac{1}{2} t_{\alpha} \sqrt{T\left(1-\varepsilon^{2}\right)}\right\} & \text { при } \varepsilon<0,
\end{array}
$$

где $t_{\alpha}-$ квантиль уровня $\alpha$ для стандартного нормального распределения вероятностей, $\Phi\left(t_{\alpha}\right)=\alpha$.

Теорема 1. Если имеет место модель вкраплений (4), то при $T \rightarrow \infty$ асимптотический размер критерия (7) проверки гипотез $H_{0, \varepsilon}, H_{1}$ на основе статистики числа серий $B_{T}$ совпадает с наперед заданным уровнем значимости $\alpha \in(0,1)$. Асимптотическое выражение мощности этого критерия для $(1,1)$-модели вкраплений и семейства простых контигуальных альтернатив $H_{1, \delta}:\left\{\delta=\frac{\rho}{T^{\beta}}\right\}, \beta>0$, имеет вид

$$
W_{1}^{B+}=W_{1}^{B-} \rightarrow\left\{\begin{array}{cc}
1, & 0<\beta<1 / 2, \\
\Phi\left(t_{\alpha}+2 \rho \frac{|\varepsilon|}{\sqrt{1-\varepsilon^{2}}}\right), & \beta=1 / 2, \\
\alpha, & \beta>1 / 2 .
\end{array}\right.
$$

Доказательство. При выполнении гипотезы $H_{0}$ по теореме Муавра-Лапласа

$$
\mathfrak{L}_{0}\left\{\frac{B_{T}-1-\frac{1}{2} T(1-\varepsilon)}{\frac{1}{2} \sqrt{T\left(1-\varepsilon^{2}\right)}}\right\} \rightarrow \mathcal{N}(0,1) \quad \text { при } \quad T \rightarrow \infty .
$$

Следовательно, при $T \rightarrow \infty$ в силу (9)

$$
\mathbf{P}_{0}\left\{\mathscr{X}_{1 \alpha}^{B+}\right\} \rightarrow \alpha, \mathbf{P}_{0}\left\{\mathscr{X}_{1 \alpha}^{B-}\right\} \rightarrow \alpha
$$

Для случая $q=r=1$ при выполнении альтернативы $H_{1}$ начальный момент 1-го порядка случайной величины $B_{T}$ в силу $(1),(2),(4),(5)$ равен

$$
\mathbf{E}_{\delta}\left\{B_{T}\right\}=1+\sum_{t=1}^{T-1} \mathbf{E}_{\delta}\left\{Y_{t} \oplus Y_{t+1}\right\}=1+2^{-1}(T-1)\left(1-(1-\delta)^{2} \varepsilon\right) .
$$


Вычислим аналогично начальный момент 2-го порядка при выполнении альтернативы $H_{1}$ :

$$
\begin{gathered}
\mathbf{E}_{\delta}\left\{B_{T}^{2}\right\}=\mathbf{E}_{\delta}\left\{\left(1+\sum_{t_{1}=1}^{T-1} Y_{t_{1}} \oplus Y_{t_{1}+1}\right)\left(1+\sum_{t_{2}=1}^{T-1} Y_{t_{2}} \oplus Y_{t_{2}+1}\right)\right\}= \\
=\mathbf{E}_{\delta}\left\{\sum_{t_{1}, t_{2}=1}^{T-1}\left(Y_{t_{1}} \oplus Y_{t_{1}+1}\right)\left(Y_{t_{2}} \oplus Y_{t_{2}+1}\right)\right\}+2 \mathbf{E}_{\delta}\left\{B_{T}\right\}-1= \\
=3 \mathbf{E}_{\delta}\left\{B_{T}\right\}-2+2 \sum_{t=1}^{T-2} \sum_{h \in V} \mathbf{P}_{\delta}\left\{Y_{t}=h, Y_{t+1}=1-h, Y_{t+2}=h\right\}+ \\
+2 \sum_{\tau=2}^{T-2} \sum_{t=1}^{T-\tau-1} \sum_{h_{1}, h_{2} \in V} \mathbf{P}_{\delta}\left\{Y_{t}=h_{1}, Y_{t+1}=1-h_{1}, Y_{t+\tau}=h_{2}, Y_{t+\tau}=1-h_{2}\right\}= \\
=3 \mathbf{E}_{\delta}\left\{B_{T}\right\}-2+2^{-1}(T-2)\left(1+\varepsilon(\varepsilon-2)(1-\delta)^{2}\right)+ \\
+4 \sum_{\tau=2}^{T-2}(T-\tau-1)\left(\mathbf{P}_{\delta}\left\{Y_{t}=0, Y_{t+1}=1, Y_{t+\tau}=0, Y_{t+\tau+1}=1\right\}+\right. \\
\left.+\mathbf{P}_{\delta}\left\{Y_{t}=0, Y_{t+1}=1, Y_{t+\tau}=1, Y_{t+\tau+1}=0\right\}\right)= \\
=1+2^{-1} 3(T-1)\left(1-\varepsilon(1-\delta)^{2}\right)+2^{-1}(T-2)\left(1+\varepsilon(\varepsilon-2)(1-\delta)^{2}\right)+ \\
+2^{-2}(T-2)(T-3)\left(1+\varepsilon\left(\varepsilon \delta^{2}-2 \varepsilon \delta-2+\varepsilon\right)(1-\delta)^{2}\right) .
\end{gathered}
$$

Находим дисперсию:

$$
\begin{gathered}
\mathbf{D}_{\delta}\left\{B_{T}\right\}= \\
=\frac{1}{4} T\left(1-(1-\delta)^{2} \varepsilon^{2}\left(1-6 \delta+3 \delta^{2}\right)-\frac{1}{4}\left(1-(1-\delta)^{2} \varepsilon^{2}\left(1-10 \delta+5 \delta^{2}\right)\right)=\right. \\
=T\left(\frac{1}{4}\left(1-(1-\delta)^{2} \varepsilon^{2}\left(1-6 \delta+3 \delta^{2}\right)\right)\right)(1+o(1)), T \rightarrow \infty .
\end{gathered}
$$

Случайная последовательность $\left\{Y_{t}\right\}$ по построению (4) удовлетворяет условию сильного перемешивания [10, 11], поэтому выполняется ЦПТ для слабозависимых случайных величин:

$$
\mathfrak{L}_{\delta}\left\{\frac{B_{T}-1-\frac{1}{2} T\left(1-(1-\delta)^{2} \varepsilon\right)}{\sqrt{\mathbf{D}_{\delta}\left\{B_{T}\right\}}}\right\} \rightarrow \mathcal{N}(0,1) .
$$

В случае $\varepsilon>0$, учитывая (10), подставим $\delta=\frac{\rho}{T^{\beta}}$ при $T \rightarrow \infty$ в выражение мощности $\lim W_{1}^{B+}=\lim \mathbf{P}_{\delta}\left\{\mathscr{X}_{1 \alpha}^{B+}\right\}=\lim \mathbf{P}_{\delta}\left\{B_{T} \geqslant 1+\frac{1}{2} T(1-\varepsilon)-\frac{1}{2} t_{\alpha} \sqrt{T\left(1-\varepsilon^{2}\right)}\right\}=$

$$
\begin{gathered}
=\lim \mathbf{P}_{\delta}\left\{\frac{B_{T}-\mathbf{E}_{\delta}\left\{B_{T}\right\}}{\sqrt{\mathbf{D}_{\delta}\left\{B_{T}\right\}}} \geqslant \frac{1+\frac{1}{2} T(1-\varepsilon)-\mathbf{E}_{\delta}\left\{B_{T}\right\}-\frac{1}{2} t_{\alpha} \sqrt{T\left(1-\varepsilon^{2}\right)}}{\sqrt{\mathbf{D}_{\delta}\left\{B_{T}\right\}}}\right\}= \\
=\Phi\left(\lim \frac{T \varepsilon \delta(2-\delta)+t_{\alpha} \sqrt{T\left(1-\varepsilon^{2}\right)}}{\sqrt{T\left(1-(1-\delta)^{2} \varepsilon^{2}\left(1-6 \delta+3 \delta^{2}\right)\right)}}\right) .
\end{gathered}
$$

Анализируя различные значения $\beta$ в этом выражении, приходим к (8). Аналогично проводятся выкладки для случая $\varepsilon<0$. 
3.2. Использование статистики коротких серий. Построим последовательность индикаторов смены состояний для последовательности $Y_{1}, \ldots, Y_{T} \in V_{T}$ :

$$
z_{t}=Y_{t} \oplus Y_{t+1} \in V, t=1, \ldots, T-1 .
$$

Определим множество шаблонов в последовательности (11):

$$
\left\{\mathfrak{b}_{1}, \mathfrak{b}_{2}, \ldots\right\}, \mathfrak{b}_{\tau}=(1, \underbrace{0, \ldots, 0}_{\tau}, 1), \tau \in \mathbb{N} \cup\{0\} \text {. }
$$

где $\mathfrak{b}_{\tau}$ - это цепочка $\tau$ подряд идущих элементов 0 , обрамленных слева и справа элементами 1. Такие шаблоны задают серии из "0" и "1" длины $\tau+1$ в стегопоследовательности $\left\{Y_{t}\right\}$. Рассмотрим несовместные случайные события $\mathfrak{C}_{\tau}, \tau \in \mathbb{N} \cup\{0\}$ :

$$
\mathfrak{C}_{\tau}=\left\{\left(z_{t}, z_{t+1}, \ldots, z_{t+\tau+1}\right)=\mathfrak{b}_{\tau}\right\}
$$

Лемма 2. Пусть имеет место модель вкраплений (4), $q=r=1$. Тогда, если справедлива альтернатива $H_{1}$, то распределение вероятностей случайных событий $\mathfrak{C}_{\tau}$ имеет вид:

$$
\mathbf{P}_{\delta}\left\{\mathfrak{C}_{\tau}\right\}=\mathbf{P}_{0}\left\{\mathfrak{C}_{\tau}\right\}+\mathfrak{a}_{\tau}(\delta, \varepsilon)=2^{-(\tau+2)}(1+\varepsilon)^{\tau}(1-\varepsilon)^{2}+\mathfrak{a}_{\tau}(\delta, \varepsilon),
$$

где $\mathfrak{a}_{\tau}(\delta, \varepsilon) \rightarrow 0$ nрu $\delta \rightarrow 0,|\varepsilon|<1$.

Доказательство. Используя формулу полной вероятности для указанной в условии модели вкраплений, получаем:

$$
\begin{gathered}
\mathbf{P}_{\delta}\left\{\mathfrak{C}_{\tau}\right\}= \\
=\sum_{u_{1}^{\tau+2} \in V_{\tau+2}} \mathbf{P}_{\delta}\left\{\gamma_{t}^{t+\tau+1}=u_{1}^{\tau+2}\right\} \mathbf{P}_{\delta}\left\{\left(z_{t}, z_{t+1}, \ldots, z_{t+\tau+1}\right)=\mathfrak{b}_{\tau} \mid \gamma_{t}^{t+\tau+1}=u_{1}^{\tau+2}\right\}= \\
=(1-\delta)^{\tau+2} \mathbf{P}_{0}\left\{\mathfrak{C}_{\tau}\right\}+\delta \sum_{u_{1}^{\tau+2} \in V_{\tau+2}: w\left(u_{1}^{\tau+2}\right)>0} \delta^{w\left(u_{1}^{\tau+2}\right)-1}(1-\delta)^{\tau+2-w\left(u_{1}^{\tau+2}\right)} \times \\
\times \mathbf{P}_{\delta}\left\{\left(z_{t}, z_{t+1}, \ldots, z_{t+\tau+1}\right)=\mathfrak{b}_{\tau} \mid \gamma_{t}^{t+\tau+1}=u_{1}^{\tau+2}\right\} \underset{\delta \rightarrow 0}{\longrightarrow} 2^{-(\tau+2)}(1+\varepsilon)^{\tau}(1-\varepsilon)^{2} .
\end{gathered}
$$

Теорема 2. В условиях леммы 2 для функиии $\mathfrak{a}_{\tau}(\delta, \varepsilon)$ справедливо асимптотическое разложение

$$
\begin{gathered}
\mathfrak{a}_{\tau}=\delta \mathfrak{a}_{\tau}^{(1)}(\varepsilon)+\mathcal{O}\left(\delta^{2}\right), \\
\mathfrak{a}_{\tau}^{(1)}(\varepsilon)= \begin{cases}2^{-1} \varepsilon(2-\varepsilon), & \tau=0 \\
2^{-2} \varepsilon(1-\varepsilon)(1+\varepsilon), & \tau=1 \\
2^{-\tau-1} \varepsilon(1-\varepsilon)(1+\varepsilon)^{\tau-2}\left(\varepsilon^{2}+(\tau+1) \varepsilon-\tau+2\right), & \tau \geqslant 2 .\end{cases}
\end{gathered}
$$

Доказательство. Разобьем множество $\mathfrak{U}_{\tau+2,1}=\left\{u_{1}^{\tau+2}=\left(u_{1}, \ldots, u_{\tau+2}\right) \in\right.$ $\left.V_{\tau+2}: w\left(u_{1}^{\tau+2}\right)=1\right\},|\mathfrak{U}|=\tau+2$, двоичных векторов длины $\tau+2, \tau \geqslant 3$, с весом Хэмминга, равным 1, на три непересекающихся подмножества:

$$
\begin{aligned}
& \mathfrak{U}_{\tau+2,1}=\mathfrak{U}_{\tau+2,1}^{(0)} \cup \mathfrak{U}_{\tau+2,1}^{(1)} \cup \mathfrak{U}_{\tau+2,1}^{(2)}, \\
& \mathfrak{U}_{\tau+2,1}^{(j)}=\left\{u_{1}^{\tau+2} \in \mathfrak{U}_{\tau+2,1}: u_{j+1}+u_{\tau+2-j}=1\right\}, j \in\{0,1\}, \\
& \mathfrak{U}_{\tau+2,1}^{(2)}=\left\{u_{1}^{\tau+2} \in \mathfrak{U}_{\tau+2,1}: \sum_{j=3}^{\tau} u_{j}=1\right\} .
\end{aligned}
$$

5 Дискретная математика, т. 27 № 3 
Используя доказательство леммы 2, имеем:

$$
\begin{gathered}
\mathbf{P}_{\delta}\left\{\mathfrak{C}_{\tau}\right\}=\mathbf{P}_{0}\left\{\mathfrak{C}_{\tau}\right\}-\delta(\tau+2) \mathbf{P}_{0}\left\{\mathfrak{C}_{\tau}\right\}+ \\
+\delta \sum_{j \in\{0,1,2\}} \sum_{u_{1}^{\tau+2} \in \mathfrak{U}_{\tau+2,1}^{(j)}} \mathbf{P}_{\delta}\left\{\left(z_{t}, z_{t+1}, \ldots, z_{t+\tau+1}\right)=\mathfrak{b}_{\tau} \mid \gamma_{t}^{t+\tau+1}=u_{1}^{\tau+2}\right\}+\mathcal{O}\left(\delta^{2}\right) .
\end{gathered}
$$

Рассмотрим случай $\tau \geqslant 2$. Подмножество $\mathfrak{U}_{\tau+2,1}^{(j)}, j \in\{0,1,2\}$, содержит такие последовательности $u_{1}^{\tau+2} \in \mathfrak{U}_{\tau+2,1}$, что события $\mathfrak{C}_{\tau} \cap\left\{\gamma_{t}^{t+\tau+1}=u_{1}^{\tau+2}\right\}$ при выполнении альтернативы $H_{1}$ равновероятны:

$$
\begin{gathered}
\mathbf{P}_{\delta}\left\{\mathfrak{C}_{\tau} \cap\left\{\gamma_{t}^{t+\tau+1}=u_{1}^{\tau+2}\right\}\right\}= \\
= \begin{cases}\delta(1-\delta)^{\tau+2} 2^{-\tau-3}(1-\varepsilon)(1+\varepsilon)^{\tau}, & u_{1}^{\tau+2} \in \mathfrak{U}_{\tau+2,1}^{(0)}, \\
\delta(1-\delta)^{\tau+2} 2^{-\tau-3}(1-\varepsilon)^{2}(1+\varepsilon)^{\tau}, & u_{1}^{\tau+2} \in \mathfrak{U}_{\tau+2,1}^{(1)}, \\
\delta(1-\delta)^{\tau+2} 2^{-\tau-3}(1-\varepsilon)^{2}\left(1+\varepsilon^{2}\right)(1+\varepsilon)^{\tau-2}, & u_{1}^{\tau+2} \in \mathfrak{U}_{\tau+2,1}^{(2)},\end{cases}
\end{gathered}
$$

Подставляя (15) в (14), получаем (13) для $\tau \geqslant 2$. Случай $\tau<2$ в (13) доказывается аналогично.

Теорема 3. В условиях леммы 2 для функиии $\mathfrak{a}_{\tau}(\delta, \varepsilon)$ справедливо асимптотическое разложение 2-го порядка:

$$
\mathfrak{a}_{\tau}=\delta \mathfrak{a}_{\tau}^{(1)}(\varepsilon)+\delta^{2} \mathfrak{a}_{\tau}^{(2)}(\varepsilon)+\mathcal{O}\left(\delta^{3}\right),
$$

где

$$
\begin{aligned}
\mathfrak{a}_{0}^{(2)}(\varepsilon)= & 2^{-2} \varepsilon(-2+\varepsilon), \mathfrak{a}_{1}^{(2)}(\varepsilon)=2^{-3} \varepsilon\left(-1+4 \varepsilon+\varepsilon^{2}\right) \\
\mathfrak{a}_{2}^{(2)}(\varepsilon)= & 2^{-4} \varepsilon^{2}\left(-7+10 \varepsilon+\varepsilon^{2}\right), \mathfrak{a}_{3}^{(2)}(\varepsilon)=2^{-5} \varepsilon\left(1-12 \varepsilon+2 \varepsilon^{2}+16 \varepsilon^{3}+\varepsilon^{4}\right), \\
\mathfrak{a}_{\tau}^{(2)}(\varepsilon)= & 2^{-\tau-2} \varepsilon(1+\varepsilon)^{\tau-4}\left(\tau-2+\varepsilon\left(2 \tau^{2}-14 \tau+13\right)+2 \varepsilon^{2}\left(-2 \tau^{2}+7 \tau-8\right)+\right. \\
& \left.+2 \varepsilon^{3}\left(\tau^{2}-3 \tau+9\right)+\varepsilon^{4}(5 \tau+2)+\varepsilon^{5}\right), \tau \geqslant 4 .
\end{aligned}
$$

Доказательство. Проводится аналогично доказательству теоремы 2 с разбиением множества стегоключей $\mathfrak{U}_{\tau+2,2}$, соответствующим разбиению на классы равновероятных событий.

Из теорем 2, 3 видно, что при выполнении альтернативы $H_{1}$ (появлении вкраплений) распределение вероятностей числа серий заданной длины изменяется. В частности, при $\varepsilon>0$ вероятности событий $\mathfrak{C}_{0}, \mathfrak{C}_{1}, \mathfrak{C}_{2}$ увеличиваются при увеличении $\delta$ от 0 до 1 , а при $\tau>\tau_{\varepsilon}=2+\varepsilon(3+\varepsilon)(1-\varepsilon)^{-1}$ вероятность $\mathbf{P}_{\delta}\left\{\mathfrak{C}_{\tau}\right\}$ уменьшается при увеличении $\delta$. Поэтому, рассмотрим статистики

$$
\mathcal{B}_{T, 1}=\sum_{t=1}^{T-2} z_{t}, \mathcal{B}_{T, 2}=\sum_{t=1}^{T-2} z_{t} z_{t+1},
$$

где статистика $\mathcal{B}_{T, 2}$ есть общее число серий из “0” и из "1" длины 1 в последовательности $\left\{y_{t}\right\}$, а статистика $\mathcal{B}_{T, 1}$ связана со статистикой числа серий $B_{T}$ соотношением $\mathcal{B}_{T, 1}=B_{T}-z_{T-1}-1$. 
Используя теорему 1 из [5], можно показать, что при выполнении альтернативы $H_{1}$ начальные моменты 1-го порядка для двумерной статистики $\left(\mathcal{B}_{T, 1}, \mathcal{B}_{T, 2}\right)$, определяемой (17), имеют вид

$$
\begin{aligned}
\mathbf{E}_{\delta}\left\{\mathcal{B}_{T, 1}\right\} & =(T-2) \frac{1}{2}\left(1-(1-\delta)^{2} \varepsilon\right)= \\
& =\mathbf{E}_{0}\left\{\mathcal{B}_{T, 1}\right\}+T \frac{1}{2} \delta(2-\delta) \varepsilon+o(T), T \rightarrow \infty, \\
\mathbf{E}_{\delta}\left\{\mathcal{B}_{T, 2}\right\} & =(T-2) \frac{1}{4}\left(1-(1-\delta)^{2} \varepsilon(2-\varepsilon)\right)= \\
& =\mathbf{E}_{0}\left\{\mathcal{B}_{T, 2}\right\}+T \frac{1}{4} \delta(2-\delta) \varepsilon(2-\varepsilon)+o(T), T \rightarrow \infty
\end{aligned}
$$

Из (18) видно, что при $\varepsilon>0$ среднее число знакоперемен или двух соседних знакоперемен при наличии вкраплений больше, чем при отсутствии вкраплений.

Теорема 4. Если имеет место модель вкраплений (4), то при $T \rightarrow \infty$ статистический критерий проверки гипотез $H_{0, \varepsilon}, H_{1}$ асимптотического уровня значимости $\alpha \in(0,1)$ на основе двумерной статистики (17) задается критической областъю

$$
\mathscr{X}_{1 \alpha}^{\mathcal{B}_{1,2}}=\left\{y_{1}^{T}:\left(\mathcal{B}_{T, 1}, \mathcal{B}_{T, 2}\right) \in \mathcal{D}_{1,2}\right\}
$$

где область $\mathcal{D}_{1,2}$ имеет вид

$$
\begin{gathered}
\mathcal{D}_{1,2}=\left\{\left(\mathcal{B}_{T, 1}, \mathcal{B}_{T, 2}\right):\left(\mathcal{B}_{T, 1}-T \mu_{0,1}\right) \varepsilon \geqslant 0,\left(\mathcal{B}_{T, 2}-T \mu_{0,1}^{2}\right) \varepsilon \geqslant 0\right. \\
\left.\left(\begin{array}{c}
\mathcal{B}_{T, 1}-\mu_{0,1} \\
\mathcal{B}_{T, 2}-\mu_{0,1}^{2}
\end{array}\right)^{\prime}\left(\begin{array}{cc}
\frac{(5-3 \varepsilon)(1-\varepsilon)}{16}-\frac{1-\varepsilon}{4} \\
-\frac{1-\varepsilon}{4} & \frac{1}{4}
\end{array}\right)\left(\begin{array}{c}
\mathcal{B}_{T, 1}-\mu_{0,1} \\
\mathcal{B}_{T, 2}-\mu_{0,1}^{2}
\end{array}\right) \geqslant T c_{1,2}\right\} \\
\mu_{0,1}=\frac{1}{2}(1-\varepsilon), c_{1,2}=2^{-5}\left(1-\varepsilon^{2}\right)^{2} \ln \frac{\pi-\arccos \left(2 \sqrt{\frac{1-\varepsilon}{5-3 \varepsilon}}\right)}{2 \pi \alpha}
\end{gathered}
$$

mo ecms

$$
\mathbf{P}_{0}\left\{\mathscr{X}_{1 \alpha}^{\mathcal{B}_{1,2}}\right\}=\mathbf{P}_{0}\left\{\left(\mathcal{B}_{T, 1}, \mathcal{B}_{T, 2}\right) \in \mathcal{D}_{1,2}\right\} \rightarrow \alpha
$$

Доказательство. Пользуясь тем, что при выполнении гипотезы $H_{0, \varepsilon}$ случайные величины $\left\{z_{t}\right\}$ независимы и имеют распределение Бернулли $\mathfrak{B}\left(2^{-1}(1-\varepsilon)\right)$, а слу- 
чайные величины $z_{t} z_{t+1}$ и $z_{s} z_{s+1}$ независимы при $|t-s|>1$, находим:

$$
\begin{gathered}
\mathbf{E}_{0}\left\{\mathcal{B}_{T, 1}\right\}=T \frac{1}{2}(1-\varepsilon)(1+o(1)), \mathbf{E}_{0}\left\{\mathcal{B}_{T, 2}\right\}=T \frac{1}{4}(1-\varepsilon)^{2}(1+o(1)), \\
\mathbf{D}_{0}\left\{\mathcal{B}_{T, 1}\right\}=(T-2) \mathbf{D}_{0}\left\{z_{t}\right\}=T \frac{1}{4}\left(1-\varepsilon^{2}\right)(1+o(1)), \\
\mathbf{D}_{0}\left\{\mathcal{B}_{T, 2}\right\}=(T-2) \mathbf{D}_{0}\left\{z_{t} z_{t+1}\right\}+2 \sum_{1 \leqslant t<s \leqslant T-2} \operatorname{cov}_{0}\left\{z_{t} z_{t+1}, z_{s} z_{s+1}\right\}= \\
=(T-2) \mathbf{D}_{0}\left\{z_{t} z_{t+1}\right\}+2(T-3) \operatorname{cov}_{0}\left\{z_{t} z_{t+1}, z_{t+1} z_{t+2}\right\}= \\
=(T-2)\left(\frac{1}{4}(1-\varepsilon)^{2}-\frac{1}{16}(1-\varepsilon)^{4}\right)+2(T-3)\left(\frac{1}{8}(1-\varepsilon)^{3}-\frac{1}{16}(1-\varepsilon)^{4}\right)= \\
=T \frac{1}{16}\left(1-\varepsilon^{2}\right)(1-\varepsilon)(5-3 \varepsilon)(1+o(1)), \\
\operatorname{cov}_{0}\left\{\mathcal{B}_{T, 1}, \mathcal{B}_{T, 2}\right\}=\sum_{t, s=1}^{T-2} \operatorname{cov}_{0}\left\{z_{t}, z_{s} z_{s+1}\right\}= \\
=(T-2) \operatorname{cov}_{0}\left\{z_{t}, z_{t} z_{t+1}\right\}+(T-3) \operatorname{cov}_{0}\left\{z_{t+1}, z_{t} z_{t+1}\right\}= \\
=(2 T-6)\left(\frac{1}{4}(1-\varepsilon)^{2}-\frac{1}{8}(1-\varepsilon)^{3}\right)=T \frac{1}{4}\left(1-\varepsilon^{2}\right)(1-\varepsilon)-\frac{3}{4}\left(1-\varepsilon^{2}\right)(1-\varepsilon)= \\
=T \frac{1}{4}\left(1-\varepsilon^{2}\right)(1-\varepsilon)(1+o(1)) .
\end{gathered}
$$

Так как последовательность пар $\left(z_{t}, z_{t} z_{t+1}\right) \in V_{2}$ является 1-зависимой, то случайный вектор $\frac{1}{\sqrt{T}}\left(\mathcal{B}_{T, 1}-\frac{1}{2} T(1-\varepsilon), \mathcal{B}_{T, 2}-\frac{1}{4} T(1-\varepsilon)^{2}\right)^{\prime}$ при $T \rightarrow \infty$ распределен асимптотически нормально по закону $\mathcal{N}_{2}\left((0,0)^{\prime}, \Sigma_{0}\right)$, где

$$
\Sigma_{0}=\left(1-\varepsilon^{2}\right)\left(\begin{array}{cc}
\frac{1}{4} & \frac{1-\varepsilon}{4} \\
\frac{1-\varepsilon}{4} & \frac{(5-3 \varepsilon)(1-\varepsilon)}{16}
\end{array}\right)
$$

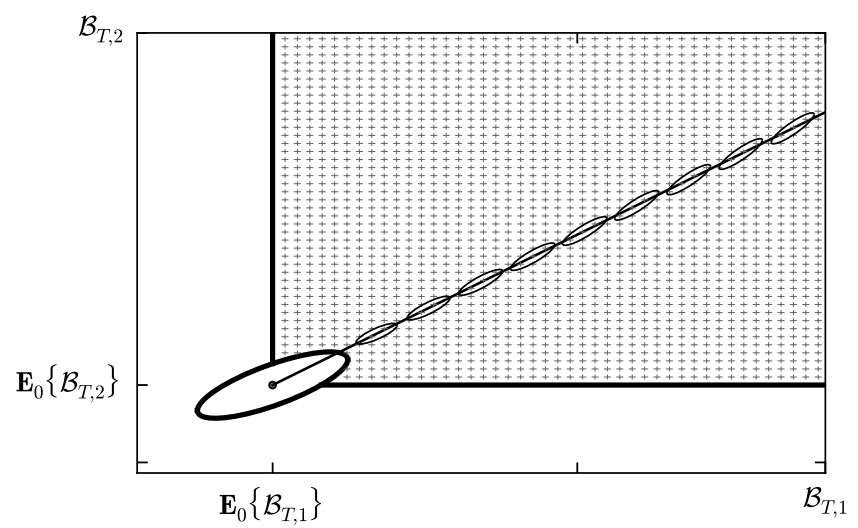

Рис. 1. Область $\mathcal{D}_{1,2}$ для случая $\varepsilon>0$ и эллипсы рассеяния при $\delta \in[0,1]$

На рис. 1 область $\mathcal{D}_{1,2}$ для случая $\varepsilon>0$ отмечена “+". Такой вид области следует из асимптотически нормального распределения двумерной статистики $\left(\mathcal{B}_{T, 1}, \mathcal{B}_{T, 2}\right)$ и выражений (18). Для вычисления вероятности ошибки первого рода осуществим линейное преобразование области $\mathcal{D}_{1,2}$. Подействуем матрицей $\Sigma_{0}^{-\frac{1}{2}}$ на единичные 
векторы $(1,0),(0,1) \in \mathcal{R}^{2}$ и построим матрицу Грама:

$$
\begin{gathered}
u_{1}=\Sigma_{0}^{-\frac{1}{2}}(1,0)^{\prime}, u_{2}=\Sigma_{0}^{-\frac{1}{2}}(0,1)^{\prime} \\
\left(\begin{array}{cc}
u_{1}^{\prime} u_{1} & u_{1}^{\prime} u_{2} \\
u_{1}^{\prime} u_{2} & u_{2}^{\prime} u_{2}
\end{array}\right)=\Sigma_{0}^{-1}=\frac{2^{6}}{\left(1-\varepsilon^{2}\right)^{2}}\left(\begin{array}{cc}
\frac{(5-3 \varepsilon)(1-\varepsilon)}{16}-\frac{1-\varepsilon}{4} \\
-\frac{1-\varepsilon}{4} & \frac{1}{4}
\end{array}\right) .
\end{gathered}
$$

Тогда угол $\phi$ между векторами $u_{1}$ и $u_{2}$ выражается через коэффициент корреляции:

$$
\phi=\arccos \left(\frac{u_{1}^{\prime} u_{2}}{\left|u_{1}\right|\left|u_{2}\right|}\right)=\pi-\arccos \left(\operatorname{corr}_{0}\left\{\mathcal{B}_{T, 1}, \mathcal{B}_{T, 2}\right\}\right)=\pi-\arccos \left(2 \sqrt{\frac{1-\varepsilon}{5-3 \varepsilon}}\right) .
$$

В силу совместной асимптотической нормальности статистик (17) случайная величина $Q_{1,2}=\frac{1}{T}\left(\begin{array}{c}\mathcal{B}_{T, 1}-\mu_{0,1} \\ \mathcal{B}_{T, 2}-\mu_{0,1}^{2}\end{array}\right)^{\prime} \Sigma_{0}^{-1}\left(\begin{array}{c}\mathcal{B}_{T, 1}-\mu_{0,1} \\ \mathcal{B}_{T, 2}-\mu_{0,1}^{2}\end{array}\right)$ при $T \rightarrow \infty$ имеет асимптотически экспоненциальное распределение с параметром $1 / 2$. Поэтому из уравнения

$$
\mathbf{P}_{0}\left\{\left(\mathcal{B}_{T, 1}, \mathcal{B}_{T, 2}\right) \in \mathcal{D}_{1,2}\right\}=\mathbf{P}_{0}\left\{Q_{1,2} \geqslant c\right\} \frac{\phi}{2 \pi}=\frac{\left(\pi-\arccos \left(2 \sqrt{\frac{1-\varepsilon}{5-3 \varepsilon}}\right)\right)}{2 \pi e^{c / 2}}=\alpha,
$$

находим $c=\frac{2^{6}}{\left(1-\varepsilon^{2}\right)^{2}} c_{1,2}$ (уравнение эллипса на рис. $1: Q_{1,2}=c$ ). Случай $\varepsilon<0$ рассматривается аналогично, при этом $u_{1}=\Sigma_{0}^{-\frac{1}{2}}(-1,0)^{\prime}, u_{2}=\Sigma_{0}^{-\frac{1}{2}}(0,-1)^{\prime}$.

Лемма 3. Для $(1,1)$-модели вкраплений при выполнении альтернативы $H_{1}$ случайные величины $z_{t}, z_{s}$ независимы при $|t-s| \geqslant 2, z_{t}, z_{s} z_{s+1}$ независимы при $|t-s| \geqslant 2$, a $z_{t} z_{t+1}, z_{s} z_{s+1}$ независимь при $|t-s| \geqslant 3$.

Доказательство. Рассмотрим случайные величины $z_{t}, z_{t+k}, k \geqslant 2$. Найдем математическое ожидание $\mathbf{E}_{\delta}\left\{z_{t} z_{t+k}\right\}, k \geqslant 2$ :

$$
\begin{gathered}
\mathbf{E}_{\delta}\left\{z_{t} z_{t+k}\right\}=\mathbf{P}_{\delta}\left\{z_{t} z_{t+k}=1\right\}=2 \mathbf{P}_{\delta}\left\{Y_{t}=0, Y_{t+1}=1, Y_{t+k}=0, Y_{t+k+1}=1\right\}+ \\
+2 \mathbf{P}_{\delta}\left\{Y_{t}=0, Y_{t+1}=1, Y_{t+k}=1, Y_{t+k+1}=0\right\}= \\
=2 \sum_{u \in V_{4}} \mathbf{P}_{\delta}\left\{\left(Y_{t}, Y_{t+1}, Y_{t+k}, Y_{t+k+1}\right)=(0,1,0,1),\left(\gamma_{t}, \gamma_{t+1}, \gamma_{t+k}, \gamma_{t+k+1}\right)=u\right\}+ \\
+2 \sum_{u \in V_{4}} \mathbf{P}_{\delta}\left\{\left(Y_{t}, Y_{t+1}, Y_{t+k}, Y_{t+k+1}\right)=(0,1,1,0),\left(\gamma_{t}, \gamma_{t+1}, \gamma_{t+k}, \gamma_{t+k+1}\right)=u\right\}= \\
=\frac{2}{16} \sum_{c \in\{1,-1\}}\left((1-\delta)^{4}(1-\varepsilon)^{2}\left(1-c \varepsilon^{k-1}\right)+\right. \\
+\delta(1-\delta)^{3}\left(2(1-\varepsilon)\left(1-c \varepsilon^{k-1}\right)+2(1-\varepsilon)\left(1+c \varepsilon^{k}\right)\right)+ \\
\left.+\delta^{2}(1-\delta)^{2}\left(6-2 \varepsilon-c \varepsilon^{k-1}+2 c \varepsilon^{k}-c \varepsilon^{k+1}\right)+4 \delta^{3}(1-\delta)+\delta^{4}\right)= \\
=\left(\frac{1}{2}\left(1-\varepsilon(1-\delta)^{2}\right)\right)^{2}=\left(\mathbf{E}_{\delta}\left\{z_{t}\right\}\right)^{2} .
\end{gathered}
$$

Так как случайные величины $z_{t}, z_{t+k}$ двоичные и $\operatorname{cov}_{\delta}\left\{z_{t}, z_{t+k}\right\}=0$ при $k \geqslant 2$, то такие величины являются попарно независимыми. Независимость для случайных величин $z_{t}, z_{s} z_{s+1}$ при $|t-s| \geqslant 2$ и $z_{t} z_{t+1}, z_{s} z_{s+1}$ при $|t-s| \geqslant 3$ доказывается аналогично. 
Найдем асимптотические выражения для моментов первого и второго порядков двумерной статистики $\left(\mathcal{B}_{T, 1}, \mathcal{B}_{T, 2}\right)$ при выполнении альтернативы $H_{1, \delta}$, используя лемму 3. Выражения для моментов первого порядка определены в (18). В ходе доказательства теоремы 1 установлено, что

$$
\mathbf{D}_{\delta}\left\{\mathcal{B}_{T, 1}\right\}=T \frac{1}{4}\left(1-(1-\delta)^{2} \varepsilon^{2}\left(1-6 \delta+3 \delta^{2}\right)\right)(1+o(1)), T \rightarrow \infty .
$$

С учетом леммы 3 при $T \rightarrow \infty$ имеем:

$$
\begin{gathered}
\operatorname{cov}_{\delta}\left\{\mathcal{B}_{T, 1}, \mathcal{B}_{T, 2}\right\}=\sum_{t, s=1}^{T-2} \operatorname{cov}_{\delta}\left\{z_{t}, z_{s} z_{s+1}\right\}= \\
=(T-2) \operatorname{cov}_{\delta}\left\{z_{t}, z_{t} z_{t+1}\right\}+(T-3) \operatorname{cov}_{\delta}\left\{z_{t}, z_{t+1} z_{t+2}\right\}+ \\
+(T-3) \operatorname{cov}_{\delta}\left\{z_{t}, z_{t-1} z_{t}\right\}+(T-4) \operatorname{cov}_{\delta}\left\{z_{t}, z_{t-2} z_{t-1}\right\}= \\
=2 T\left(\operatorname{cov}_{\delta}\left\{z_{t}, z_{t} z_{t+1}\right\}+\operatorname{cov}_{\delta}\left\{z_{t}, z_{t+1} z_{t+2}\right\}\right)(1+o(1)) \\
\operatorname{cov}_{\delta}\left\{z_{t}, z_{t} z_{t+1}\right\}=\mathbf{P}_{\delta}\left\{z_{t} z_{t+1}=1\right\}\left(1-\mathbf{P}_{\delta}\left\{z_{t}=1\right\}\right)= \\
=\frac{1}{4}\left(1-(1-\delta)^{2} \varepsilon(2-\varepsilon)\right)\left(1-\frac{1}{2}\left(1-(1-\delta)^{2} \varepsilon\right)\right)= \\
=\frac{1}{8}\left(1-(1-\delta)^{2} \varepsilon(1-\varepsilon)-(1-\delta)^{4} \varepsilon^{2}(2-\varepsilon)\right), \\
\operatorname{cov}_{\delta}\left\{z_{t}, z_{t+1} z_{t+2}\right\}=\mathbf{P}_{\delta}\left\{z_{t} z_{t+1} z_{t+2}=1\right\}-\mathbf{P}_{\delta}\left\{z_{t}=1\right\} \mathbf{P}_{\delta}\left\{z_{t+1} z_{t+2}=1\right\} .
\end{gathered}
$$

Используя формулу полной вероятности, для модели вкраплений $(1,1)$ находим

$$
\begin{gathered}
\mathbf{P}_{\delta}\left\{z_{t} z_{t+1} z_{t+2}=1\right\}=2 \mathbf{P}_{\delta}\left\{Y_{t}=0, Y_{t+1}=1, Y_{t+2}=0, Y_{t+3}=1\right\}= \\
=2 \sum_{u \in V_{4}} \mathbf{P}_{\delta}\left\{Y_{t}=0, Y_{t+1}=1, Y_{t+2}=0, Y_{t+3}=1, \gamma_{t}^{t+3}=u\right\}= \\
=\frac{1}{8}\left(1-(1-\delta)^{2} \varepsilon\left(3-2 \varepsilon+\varepsilon^{2}\right)+(1-\delta)^{4} \varepsilon^{2}\right), \\
\operatorname{cov}_{\delta}\left\{z_{t}, z_{t+1} z_{t+2}\right\}=\mathbf{P}_{\delta}\left\{z_{t} z_{t+1} z_{t+2}=1\right\}-\mathbf{P}_{\delta}\left\{z_{t}=1\right\} \mathbf{P}_{\delta}\left\{z_{t+1} z_{t+2}=1\right\}= \\
=\mathbf{P}_{\delta}\left\{z_{t} z_{t+1} z_{t+2}=1\right\}-\frac{1}{8}\left(1-(1-\delta)^{2} \varepsilon(3-\varepsilon)+(1-\delta)^{4} \varepsilon^{2}(2-\varepsilon)\right)= \\
=\frac{1}{8}(1-\delta)^{2} \delta(2-\delta) \varepsilon^{2}(1-\varepsilon) .
\end{gathered}
$$

Таким образом, получаем

$$
\operatorname{cov}_{\delta}\left\{\mathcal{B}_{T, 1}, \mathcal{B}_{T, 2}\right\}=T \frac{1}{4}\left(1-(1-\delta)^{2} \varepsilon(1-\varepsilon)^{2}-(1-\delta)^{4} \varepsilon^{2}(3-2 \varepsilon)\right)(1+o(1)) .
$$

С учетом леммы 3 при $T \rightarrow \infty$ найдем дисперсию $\mathbf{D}_{\delta}\left\{\mathcal{B}_{T, 2}\right\}$ :

$$
\begin{gathered}
\mathbf{D}_{\delta}\left\{\mathcal{B}_{T, 2}\right\}=(T-2) \mathbf{D}_{\delta}\left\{z_{t} z_{t+1}\right\}+2(T-3) \operatorname{cov}_{\delta}\left\{z_{t} z_{t+1}, z_{t+1} z_{t+2}\right\}+ \\
+2(T-4) \operatorname{cov} \delta\left\{z_{t} z_{t+1}, z_{t+2} z_{t+3}\right\} \\
\mathbf{D}_{\delta}\left\{z_{t} z_{t+1}\right\}=\mathbf{P}_{\delta}\left\{z_{t} z_{t+1}=1\right\}\left(1-\mathbf{P}_{\delta}\left\{z_{t} z_{t+1}=1\right\}=\right. \\
=\frac{1}{16}\left(1-(1-\delta)^{2} \varepsilon(2-\varepsilon)\right)\left(3+(1-\delta)^{2} \varepsilon(2-\varepsilon)\right), \\
\operatorname{cov}_{\delta}\left\{z_{t} z_{t+1}, z_{t+1} z_{t+2}\right\}=\mathbf{P}_{\delta}\left\{z_{t} z_{t+1} z_{t+2}=1\right\}-\left(\mathbf{P}_{\delta}\left\{z_{t} z_{t+1}=1\right\}\right)^{2}= \\
=\frac{1}{16}\left(1-(1-\delta)^{2} 2 \varepsilon\left(1-\varepsilon+\varepsilon^{2}\right)-(1-\delta)^{4} \varepsilon^{2}\left(2-4 \varepsilon+\varepsilon^{2}\right)\right), \\
\operatorname{cov}_{\delta}\left\{z_{t} z_{t+1}, z_{t+2} z_{t+3}\right\}=\mathbf{P}_{\delta}\left\{z_{t} z_{t+1} z_{t+2} z_{t+3}=1\right\}-\left(\mathbf{P}_{\delta}\left\{z_{t} z_{t+1}=1\right\}\right)^{2} .
\end{gathered}
$$


Аналогично нахождению $\mathbf{P}_{\delta}\left\{z_{t} z_{t+1} z_{t+2}=1\right\}$ имеем:

$$
\begin{aligned}
& \mathbf{P}_{\delta}\left\{z_{t} z_{t+1} z_{t+2} z_{t+3}=1\right\}=2 \mathbf{P}_{\delta}\left\{Y_{t}=0, Y_{t+1}=1, Y_{t+2}=0, Y_{t+3}=1, Y_{t+4}=0\right\}= \\
& =2 \sum_{u \in V_{5}} \mathbf{P}_{\delta}\left\{Y_{t}=0, Y_{t+1}=1, Y_{t+2}=0, Y_{t+3}=1, Y_{t+4}=0, \gamma_{t}^{t+4}=u\right\}= \\
& =\frac{1}{16}\left(1-(1-\delta)^{2}\left(\varepsilon\left(4+\delta^{3}\right)-3 \varepsilon^{2}\left(2-2 \delta+\delta^{2}\right)+\varepsilon^{3}\left(4-4 \delta+2 \delta^{2}-\delta^{3}\right)-\varepsilon^{4}\right)\right), \\
& \operatorname{cov}_{\delta}\left\{z_{t} z_{t+1}, z_{t+2} z_{t+3}\right\}=\frac{1}{16}(1-\delta)^{2} \delta \varepsilon(1-\varepsilon)\left(-\delta^{2}+\varepsilon\left(2-\delta-\delta^{2}\right)-\varepsilon^{2}(2-\delta)\right)
\end{aligned}
$$

Таким образом, получаем

$$
\begin{gathered}
\mathbf{D}_{\delta}\left\{\mathcal{B}_{T, 2}\right\}=T \frac{1}{16}\left(5-(1-\delta)^{2}\left(2\left(4+\delta^{3}\right) \varepsilon+2\left(1-10 \delta+5 \delta^{2}\right) \varepsilon^{2}-\right.\right. \\
\left.\left.-2\left(4-16 \delta+8 \delta^{2}+\delta^{3}\right) \varepsilon^{3}+\left(3-10 \delta+\delta^{2}\right) \varepsilon^{4}\right)\right)(1+o(1)) .
\end{gathered}
$$

Используя свойство сильного перемешивания [10], можно доказать, что для альтернативы $H_{1, \delta}$ при $T \rightarrow \infty$ случайный вектор $\frac{1}{\sqrt{T}}\left(\mathcal{B}_{T, 1}-\frac{1}{2} T\left(1-(1-\delta)^{2} \varepsilon\right), \mathcal{B}_{T, 2}-\frac{1}{4} T\left(1-(1-\delta)^{2} \varepsilon(2-\varepsilon)\right)\right)^{\prime}$ распределен асимптотически нормально по закону $\mathcal{N}_{2}\left((0,0)^{\prime}, \Sigma_{1}\right)$ с нулевым средним и ковариационной матрицей $\Sigma_{1}=\left(\sigma_{1, i j}\right), i, j=1,2$ :

$$
\begin{gathered}
\sigma_{1,00}=\frac{1}{4}\left(1-(1-\delta)^{2} \varepsilon^{2}\left(1-6 \delta+3 \delta^{2}\right)\right), \\
\sigma_{1,01}=\sigma_{1,10}=\frac{1}{4}\left(1-(1-\delta)^{2} \varepsilon(1-\varepsilon)^{2}-(1-\delta)^{4} \varepsilon^{2}(3-2 \varepsilon)\right), \\
\sigma_{1,11}=\frac{1}{16}\left(5-(1-\delta)^{2}\left(2\left(4+\delta^{3}\right) \varepsilon+2\left(1-10 \delta+5 \delta^{2}\right) \varepsilon^{2}-\right.\right. \\
\left.\left.-2\left(4-16 \delta+8 \delta^{2}+\delta^{3}\right) \varepsilon^{3}+\left(3-10 \delta+\delta^{2}\right) \varepsilon^{4}\right)\right) .
\end{gathered}
$$

Для критерия (19) на основе статистики коротких серий, к сожалению, не удается получить в явном виде выражение мощности и исследовать ее, так как ковариационная матрица зависит от $\delta$. Эта зависимость иллюстрируется рисунком 1 , на котором изображены эллипсы рассеяния (соответствующие асимптотической ковариационной матрице) при увеличении параметра $\delta$ от 0 до 1. Отметим важное свойство асимптотически нормального распределения случайного вектора (17) при альтернативе $H_{1, \delta}$ : при изменении $\delta$ от 0 до 1 центр асимптотически нормального распределения двумерной статистики $\left(\mathcal{B}_{T, 1}, \mathcal{B}_{T, 2}\right)$ неизменно лежит на прямой:

$$
\left\{\begin{array}{l}
b_{1}=\frac{1}{2} T \varepsilon \Delta+\frac{1}{2} T(1-\varepsilon), \\
b_{2}=\frac{1}{4} T \varepsilon(2-\varepsilon) \Delta+\frac{1}{4} T(1-\varepsilon)^{2}, \quad \Delta=\delta(2-\delta) .
\end{array}\right.
$$

Учитывая указанное свойство (22), построим статистический критерий проверки гипотез $H_{0, \varepsilon}, H_{1}$ на основе статистики, являющейся ортогональной проекцией статистики $\left(\mathcal{B}_{T, 1}, \mathcal{B}_{T, 2}\right)$ на прямую $(22)$. Такой критерий при $\varepsilon>0$ задается критической областью

$$
\begin{gathered}
\mathscr{X}_{1 \alpha}^{\mathfrak{h}+}=\left\{y_{1}^{T}: \mathcal{B}_{T, 1}+\frac{1}{2}(2-\varepsilon) \mathcal{B}_{T, 2} \geqslant \frac{1}{2} T(1-\varepsilon)+\frac{1}{8} T(1-\varepsilon)^{2}(2-\varepsilon)-t_{\alpha} \sqrt{T d_{\mathfrak{h}}}\right\}, \\
d_{\mathfrak{h}}=2^{-6}\left(1-\varepsilon^{2}\right)\left(68-100 \varepsilon+65 \varepsilon^{2}-20 \varepsilon^{3}+3 \varepsilon^{4}\right) .
\end{gathered}
$$


Теорема 5. Если имеет место модель вкраплений (4) и $\varepsilon>0$, то при $T \rightarrow \infty$ асимптотический размер критерия (23) проверки гипотез $H_{0, \varepsilon}, H_{1}$ на основе проекции статистики коротких серий

$$
\mathfrak{h}=\mathcal{B}_{T, 1}-\frac{1}{2} T(1-\varepsilon)+\frac{1}{2}(2-\varepsilon)\left(\mathcal{B}_{T, 2}-\frac{1}{4} T(1-\varepsilon)^{2}\right)
$$

совпадает с уровнем значимости $\alpha \in(0,1)$. Асимптотическая мощность этого критерия для $(1,1)$-модели вкраплений и семейства контигуальных альтернатив $H_{1, \delta}:\left\{\delta=\frac{\rho}{\sqrt{T}}\right\}$ имеет вид

$$
W_{1}^{\mathfrak{h}+} \rightarrow \Phi\left(t_{\alpha}+\frac{\rho \varepsilon\left(1+\frac{1}{4}(2-\varepsilon)^{2}\right)}{\sqrt{d_{\mathfrak{h}}}}\right), T \rightarrow \infty .
$$

Доказательство. Угол между прямой $(22)$ и осью $b_{2}$ равен $\phi=\arctan \left(\frac{1}{2}(2-\varepsilon)\right)$, поэтому ортогональная проекция точки $\left(\mathcal{B}_{T, 1}, \mathcal{B}_{T, 2}\right)$ на эту прямую есть

$$
\left(\mathcal{B}_{T, 1}-\frac{1}{2} T(1-\varepsilon)\right) \cos \phi+\left(\mathcal{B}_{T, 1}-\frac{1}{2} T(1-\varepsilon)\right) \sin \phi .
$$

Умножив это выражение на $\operatorname{cosec} \phi$, получаем случайную величину $\mathfrak{h}$, которая согласно (21) при выполнении гипотезы $H_{0, \varepsilon}$ имеет асимптотически нормальное распределение $\mathcal{N}_{1}\left(0, d_{\mathfrak{h}}\right)$. Следовательно, $\mathbf{P}_{0}\left\{\mathscr{X}_{1 \alpha}^{\mathfrak{h}+}\right\} \rightarrow \alpha$ при $T \rightarrow \infty$.

Найдем мощность критерия (23) при $T \rightarrow \infty$ для контигуальных альтернатив вида, указанного в формулировке теоремы:

$$
\begin{gathered}
W_{1}^{\mathfrak{h}+}=\mathbf{P}_{\delta}\left\{\mathcal{B}_{T, 1}+\frac{1}{2}(2-\varepsilon) \mathcal{B}_{T, 2} \geqslant \frac{1}{2} T(1-\varepsilon)+\frac{1}{8} T(1-\varepsilon)^{2}(2-\varepsilon)-t_{\alpha} \sqrt{T d_{\mathfrak{h}}}\right\} \\
=\mathbf{P}_{\delta}\left\{\mathcal{B}_{T, 1}+\frac{1}{2}(2-\varepsilon) \mathcal{B}_{T, 2}-\mathbf{E}_{\delta}\left\{\mathcal{B}_{T, 1}\right\}-\frac{1}{2}(2-\varepsilon) \mathbf{E}_{\delta}\left\{\mathcal{B}_{T, 2}\right\} \leqslant\right. \\
\left.\leqslant \frac{1}{2} T \delta(2-\delta) \varepsilon+\frac{1}{8} T \delta(2-\delta) \varepsilon(2-\varepsilon)^{2}+t_{\alpha} \sqrt{T d_{\mathfrak{h}}}\right\} \rightarrow \\
\rightarrow \Phi\left(\lim \frac{\left.\frac{1}{2} T \delta(2-\delta) \varepsilon+\frac{1}{8} T \delta(2-\delta) \varepsilon(2-\varepsilon)^{2}+t_{\alpha} \sqrt{T d_{\mathfrak{h}}}\right\}}{\sqrt{T\left(\sigma_{1,00}+\frac{1}{4}(2-\varepsilon)^{2} \sigma_{1,11}+(2-\varepsilon) \sigma_{1,01}\right)}}\right)
\end{gathered}
$$

Подставляя в это выражение $\delta=\frac{\rho}{\sqrt{T}}$ при $T \rightarrow \infty$, получим

$$
\begin{gathered}
W_{1}^{\mathfrak{h}+} \rightarrow \Phi\left(\lim \frac{\sqrt{T} \rho \varepsilon+\frac{1}{4} \sqrt{T} \rho \varepsilon(2-\varepsilon)^{2}+t_{\alpha} \sqrt{T d_{\mathfrak{h}}}+\mathcal{O}(1)}{\sqrt{T\left(d_{\mathfrak{h}}+\mathcal{O}\left(\frac{1}{\sqrt{T}}\right)\right)}}\right) \rightarrow \\
\rightarrow \Phi\left(t_{\alpha}+\frac{\rho \varepsilon\left(1+\frac{1}{4}(2-\varepsilon)^{2}\right)}{\sqrt{d_{\mathfrak{h}}}}\right) .
\end{gathered}
$$

\section{4. Обнаружение вкраплений на основе статистики отношения правдоподобия}

Рассмотрим теперь случай, когда параметр $\varepsilon$ в (1) неизвестен и отделен от нуля: $\varepsilon_{0} \leqslant|\varepsilon|<1$, где $\varepsilon_{0}>0$ - известное граничное значение. 
Построим функцию правдоподобия для наблюдаемой стегопоследовательности $y_{1}^{T} \in V_{T}$. Следуя [5], разобьем множество $V_{t}$ двоичных $t$-мерных векторов на $t+1$ непересекающихся подмножеств:

$$
V_{t}=\Gamma_{0}^{(t)} \cup \Gamma_{1}^{(t)} \cup \ldots \cup \Gamma_{t}^{(t)},
$$

где

$$
\begin{aligned}
& \Gamma_{0}^{(t)}=\left\{u_{1}^{t} \in V_{t}: u_{t}=1\right\}, \\
& \Gamma_{1}^{(t)}=\left\{u_{1}^{t} \in V_{t}: u_{t-1}=u_{t}=0\right\}, \\
& \Gamma_{j}^{(t)}=\left\{u_{1}^{t} \in V_{t}: u_{t-j}=0, u_{t-j+1}=\ldots=u_{t-1}=1, u_{t}=0\right\}, 1<j<t, \\
& \Gamma_{t}^{(t)}=\left\{u_{1}^{t} \in V_{t}: u_{1}=\ldots=u_{t-1}=u_{t}=1\right\} .
\end{aligned}
$$

Разбиение (26), (27) порождает разбиение всевозможных траекторий фрагментов ключевой последовательности $\gamma_{1}^{t}=u_{1}^{t} \in V_{t}$.

Лемма 4. Функция правдоподобия для $(q, r)$-блочной модели вкраплений имеет вид

$$
L(\varepsilon, \delta)=\mathbf{P}_{\delta}\left\{Y_{1}^{T}=y_{1}^{T}\right\}=2^{-T} \sum_{u_{1}^{T} \in \Gamma^{(q, r)}}(1-\delta)^{b_{0}\left(u_{1}^{T}\right)}\left(\delta / C_{q}^{r}\right)^{b_{r}\left(u_{1}^{T}\right)} \prod_{t=1}^{T} \varphi_{t}\left(u_{1}^{t}, y_{1}^{t}\right),
$$

где

$$
\varphi_{t}\left(u_{1}^{t}, y_{1}^{t}\right)=\left\{\begin{array}{l}
1, u_{1}^{t} \in \Gamma_{0}^{(t)}, \\
1+(-1)^{y_{t-j}+y_{t}} \varepsilon^{j}, u_{1}^{t} \in \Gamma_{j}^{(t)}, 1 \leqslant j<t, \\
1, u_{1}^{t} \in \Gamma_{t}^{(t)} .
\end{array}\right.
$$

Доказательство. Проводится аналогично доказательству теоремы 5 для $q$-блочной модели вкраплений в [5].

Для проверки гипотез $H_{0}, H_{1}$ о наличии и отсутствии вкраплений построим теперь статистический критерий отношения правдоподобия [12]. Статистика $\lambda_{T}$ этого критерия для проверки гипотез $H_{0}, H_{1}$ имеет вид

$$
\lambda_{T}=\lambda_{T}\left(y_{1}^{T}\right)=-2 \ln \frac{L(\hat{\varepsilon}, 0)}{\max \left\{L\left(\hat{\varepsilon}_{1}, \hat{\delta}_{1}\right), L(\hat{\varepsilon}, 0)\right\}} \geqslant 0,
$$

где $\hat{\varepsilon},\left(\hat{\varepsilon}_{1}, \hat{\delta}_{1}\right)$ - МП-оценки, построенные в [5], соответственно при условиях $H_{0}$ и $H_{1}$. Введенная статистика (28) эквивалентна статистике отношения правдоподобия $\sup \mathbf{P}_{\delta}\left\{y_{1}, \ldots, y_{T}\right\}$

$\frac{|\varepsilon|<1, \delta>0}{\sup \mathbf{P}_{0}\left\{y_{1}, \ldots, y_{T}\right\}}$, причем, как установлено в [5],

$|\varepsilon|<1$

$$
\arg \max _{|\varepsilon|<1, \delta>0} \mathbf{P}_{\delta}\left\{y_{1}, \ldots, y_{T}\right\}=\left(\hat{\varepsilon}_{1}, \hat{\delta}_{1}\right), \arg \max _{|\varepsilon|<1} \mathbf{P}_{0}\left\{y_{1}, \ldots, y_{T}\right\}=\hat{\varepsilon}
$$

Статистический критерий размера $\alpha \in(0,1)$ на основе статистики $\lambda_{T}$ задается критической областью

$$
\mathscr{X}_{1 \alpha}^{\lambda}=\left\{y_{1}^{T} \in V_{T}: \lambda_{T} \geqslant \lambda_{\alpha}\right\}
$$


где $\lambda_{\alpha}>0$ есть решение уравнения

$$
\sup _{\varepsilon_{0} \leqslant|\varepsilon|<1} \mathbf{P}_{0}\left\{\lambda_{T} \geqslant \lambda\right\}=\sup _{\varepsilon_{0} \leqslant|\varepsilon|<1}\left(1-F_{0}\left(\varepsilon, T, \lambda_{T}\right)\right)=\alpha .
$$

Здесь $F_{0}\left(\varepsilon, T, \lambda_{T}\right)$ - функция распределения статистики (28) при нулевой гипотезе $H_{0}$.

Для оценки значения $\lambda_{\alpha}$, удовлетворяющего (30), применим метод Монте-Карло: моделируем $M_{0}$ реализаций цепи Маркова длины $T$ с параметром $\varepsilon_{0}$. Для каждой реализации вычисляем значение статистики, определяемой (28). Обозначим вычисленные значения $\lambda^{(1)}, \ldots, \lambda^{\left(M_{0}\right)}$. Тогда $\lambda_{\alpha}$ можно оценить выборочной квантилью уровня $1-\alpha$ :

$$
\hat{\lambda}_{\alpha}=\lambda_{\left(\left[(1-\alpha) M_{0}\right]\right)}
$$

точность этой оценки возрастает при $M_{0} \rightarrow \infty$. Таким образом, статистический критерий (29) проверки гипотез о наличии вкраплений примет вид:

$$
\begin{gathered}
\text { принимается гипотеза }\left\{\begin{array}{l}
H_{0}, \text { если } p \geqslant \alpha, \\
H_{1}, \text { если } p<\alpha,
\end{array},\right. \\
p=\frac{1}{M_{0}+1}\left(1+\sum_{i=1}^{M_{0}} I\left\{\lambda^{(i)}>\lambda_{T}\right\}\right) .
\end{gathered}
$$

Если выполняются условия регулярности [12], обеспечивающие существование, единственность и асимптотическую нормальность оценок максимального правдоподобия параметров $\varepsilon, \delta$, то можно использовать известные асимптотические свойства критерия отношения правдоподобия $[12,13]$.

Теорема 6. Пусть имеет место модель вкраплений (4), тогда при $T \rightarrow \infty$ критерий асимптотического уровня значимости $\alpha \in(0,1)$ на основе статистики отношения правдоподобия для сложной нулевой гипотезы задается критической областъю (29), где порог $\lambda_{\alpha}=\chi_{1-\alpha, 1}^{2}$, то есть

$$
\mathbf{P}_{0}\left\{\mathscr{X}_{1 \alpha}^{\lambda}\right\}=\mathbf{P}_{0}\left\{\lambda_{T} \geqslant \chi_{1-\alpha, 1}^{2}\right\} \rightarrow \alpha
$$

этот критерий является состоятельным при фиксированных альтернативах $\delta=$ $\delta_{1}>0$ :

$$
W_{1}^{\lambda}=\mathbf{P}_{\delta}\left\{\mathscr{X}_{1 \alpha}^{\lambda}\right\} \rightarrow 1
$$

Доказательство. Проводится по схеме из [13] с использованием центральной предельной теоремы для слабозависимых случайных величин [10].

\section{5. Статистическое оценивание точек вкраплений}

Если принимается альтернатива $H_{1}$, то возникает задача оценивания точек вкраплений, т.е. моментов времени $t \in\{1, \ldots, T\}$, для которых согласно (4) бит последовательности $\left\{x_{t}\right\}$ заменяется на бит скрытого сообщения $\left\{\xi_{t}\right\}$. 
Теорема 7. Пусть $\gamma_{1}^{T}=\left(\gamma_{1}, \ldots, \gamma_{T}\right) \in \Gamma^{(q, r)}-$ ключевая последовательность, соответствующая $(q, r)$-модели вкраплений, $y_{1}^{T} \in V_{T}$ - наблюдаемая стегопоследовательность, $\hat{\gamma}_{1}^{T}=f\left(y_{1}^{T}\right)$ - некоторая статистическая оценка ключевой последовательности $\gamma_{1}^{T}$ по наблюдениям $y_{1}^{T}$. Минимум вероятности ошибки оценивания стегоключа

$$
\mathbf{P}_{\delta}\left\{\hat{\gamma}_{1}^{T} \neq \gamma_{1}^{T}\right\} \rightarrow \min
$$

достигается для статистики

$$
\hat{\gamma}_{1}^{T *}=\arg \max _{u_{1}^{T} \in \Gamma^{(q, r)}} \mathbf{P}_{\delta}\left\{\gamma_{1}^{T}=u_{1}^{T} \mid Y_{1}^{T}=y_{1}^{T}\right\},
$$

максимизирующей апостериорную вероятность стегоключа; при этом минимальное возможное значение вероятности ошибки равно

$$
\begin{gathered}
r^{*}(\varepsilon, \delta, T)=\min _{f(\cdot)} \mathbf{P}_{\delta}\left\{\hat{\gamma}_{1}^{T} \neq \gamma_{1}^{T}\right\}= \\
=1-\sum_{y_{1}^{T} \in V_{T}} \mathbf{P}_{\delta}\left\{Y_{1}^{T}=y_{1}^{T}\right\} \max _{u_{1}^{T} \in \Gamma^{(q, r)}} \mathbf{P}_{\delta}\left\{\gamma_{1}^{T}=u_{1}^{T} \mid Y_{1}^{T}=y_{1}^{T}\right\} .
\end{gathered}
$$

Доказательство. Выберем произвольную статистику

$$
\hat{\gamma}_{1}^{T}=f\left(Y_{1}^{T}\right): V_{T} \rightarrow \Gamma^{(q, r)},
$$

и вычислим для нее вероятность ошибки при оценивании истинного стегоключа $\gamma_{1}^{T} \in \Gamma^{(q, r)}:$

$$
r(f ; \varepsilon, \delta, T)=\mathbf{P}_{\delta}\left\{\hat{\gamma}_{1}^{T} \neq \gamma_{1}^{T}\right\}=1-\mathbf{P}_{\delta}\left\{\hat{\gamma}_{1}^{T}=\gamma_{1}^{T}\right\} .
$$

Проводя эквивалентные преобразования с учетом (34) и формулы полной вероятности, получим

$$
\begin{aligned}
r(f ; \varepsilon, \delta, T) & =1-\sum_{u_{1}^{T} \in \Gamma^{(q, r)}} \mathbf{P}_{\delta}\left\{\hat{\gamma}_{1}^{T}=\gamma_{1}^{T}, \gamma_{1}^{T}=u_{1}^{T}\right\}= \\
& =1-\sum_{u_{1}^{T} \in \Gamma^{(q, r)}} \sum_{y_{1}^{T} \in V_{T}} \mathbf{P}_{\delta}\left\{f\left(Y_{1}^{T}\right)=\gamma_{1}^{T}, \gamma_{1}^{T}=u_{1}^{T}, Y_{1}^{T}=y_{1}^{T}\right\}= \\
= & 1-\sum_{y_{1}^{T} \in V_{T}} \sum_{u_{1}^{T} \in \Gamma^{(q, r)}} \mathbf{P}_{\delta}\left\{Y_{1}^{T}=y_{1}^{T}\right\} \mathbf{P}_{\delta}\left\{\gamma_{1}^{T}=u_{1}^{T} \mid Y_{1}^{T}=y_{1}^{T}\right\} \times \\
& \times \mathbf{P}_{\delta}\left\{f\left(Y_{1}^{T}\right)=\gamma_{1}^{T} \mid \gamma_{1}^{T}=u_{1}^{T}, Y_{1}^{T}=y_{1}^{T}\right\}= \\
= & =\sum_{y_{1}^{T} \in V_{T}} \mathbf{P}_{\delta}\left\{Y_{1}^{T}=y_{1}^{T}\right\} \sum_{u_{1}^{T} \in \Gamma^{(q, r)}} I\left\{f\left(y_{1}^{T}\right)=u_{1}^{T}\right\} \mathbf{P}_{\delta}\left\{\gamma_{1}^{T}=u_{1}^{T} \mid Y_{1}^{T}=y_{1}^{T}\right\} .
\end{aligned}
$$

Минимизируя это выражение по $f(\cdot)$ согласно (34), получаем оптимальную функцию $f(\cdot)$ в следующем виде:

$$
f^{*}\left(y_{1}^{T}\right)=\arg \max _{u_{1}^{T} \in \Gamma^{(q, r)}} \mathbf{P}_{\delta}\left\{\gamma_{1}^{T}=u_{1}^{T} \mid Y_{1}^{T}=y_{1}^{T}\right\}
$$

что совпадает со статистикой (32).

Подставляя (36) в (35), получаем (33). 
Оценка (32) по методу максимума апостериорной вероятности допускает следующее эквивалентное представление, удобное для ее вычисления:

$$
\begin{gathered}
\hat{\gamma}_{1}^{T *}=\arg \max _{u_{1}^{T} \in \Gamma^{(q, r)}} \mathbf{P}_{\delta}\left\{\gamma_{1}^{T}=u_{1}^{T} \mid Y_{1}^{T}=y_{1}^{T}\right\}= \\
=\arg \max _{u_{1}^{T} \in \Gamma^{(q, r)}} \mathbf{P}_{\delta}\left\{\gamma_{1}^{T}=u_{1}^{T}, Y_{1}^{T}=y_{1}^{T}\right\} .
\end{gathered}
$$

Решение задачи (37) для $(q, r)$-блочной модели вкраплений прямым перебором имеет вычислительную сложность порядка $O\left(T\left(1+C_{q}^{r}\right)^{T / q}\right)$. Построим полиномиальный алгоритм решения этой задачи, основанный на классическом алгоритме Витерби [14].

Обозначим:

$$
\begin{gathered}
\mathfrak{s}_{t}\left(u_{t-c}, \ldots, u_{t}\right)=\max _{u_{1}, \ldots, u_{t-c-1} \in V} \log \mathbf{P}_{\delta}\left\{Y_{1}^{t}=y_{1}^{t}, \gamma_{1}=u_{1}, \ldots, \gamma_{t}=u_{t}\right\} \\
c=\max \{2 r+1, q-1\} .
\end{gathered}
$$

Начальные значения $\mathfrak{s}_{t}\left(u_{1}, \ldots, u_{t}\right)$ при $t=1, \ldots, c$ определяются следующим обра3ом:

$$
\begin{aligned}
\mathfrak{s}_{1}\left(u_{1}\right)= & \log \varphi_{1}\left(u_{1}, y_{1}\right)+\log \mathbf{P}_{\delta}\left\{\gamma_{1}=u_{1}\right\}, \\
\mathfrak{s}_{t}\left(u_{1}, \ldots, u_{t}\right)= & \mathfrak{s}_{t-1}\left(u_{1}, \ldots, u_{t-1}\right)+\log \varphi_{t}\left(u_{1}^{t}, y_{1}^{t}\right)+ \\
& +\log \mathbf{P}_{\delta}\left\{\gamma_{t}=u_{t} \mid \gamma_{t-1}=u_{t-1}, \ldots, \gamma_{1}=u_{1}\right\}, 2 \leqslant t \leqslant c,
\end{aligned}
$$

где $\varphi_{t}(\cdot)$ найдена в лемме 4.

Теорема 8. Если имеет место $(q, r)$-блочная модель вкраплений $(4), q>r$, то для $\mathfrak{s}_{t}\left(u_{t-c}, \ldots, u_{t}\right)$ при $t>c$ справедливо рекуррентное соотношение:

$$
\begin{gathered}
\mathfrak{s}_{t}\left(u_{t-c}, \ldots, u_{t}\right)= \\
=\max _{u_{t-c-1} \in V} \mathfrak{s}_{t-1}\left(u_{t-c-1}, u_{t-c}, \ldots, u_{t-1}\right)+\log \mathfrak{f}_{t}\left(u_{t-2 r-1}^{t}, y_{t-2 r-1}^{t}\right)+ \\
\quad+\log \mathbf{P}_{\delta}\left\{\gamma_{t}=u_{t} \mid \gamma_{t-1}=u_{t-1}, \ldots, \gamma_{t-c}=u_{t-c}\right\}
\end{gathered}
$$

где

$$
\mathfrak{f}_{t}\left(u_{t-2 r-1}^{t}, y_{t-2 r-1}^{t}\right)=\left\{\begin{array}{l}
\frac{1}{2}, u_{1}^{t} \in \Gamma_{0}^{(t)}, \\
\frac{1}{2}\left(1+(-1)^{y_{t-j}+y_{t}} \varepsilon^{j}\right), u_{1}^{t} \in \Gamma_{j}^{(t)}, 1 \leqslant j \leqslant 2 r+1 .
\end{array}\right.
$$

Доказательство. В случае $q \leqslant 2 r+2$ имеем цепочку равенств:

$$
\begin{gathered}
\mathfrak{s}_{t}\left(u_{t-2 r-1}, \ldots, u_{t}\right)=\max _{u_{1}, \ldots, u_{t-2 r-2} \in V} \log \mathbf{P}_{\delta}\left\{Y_{1}^{t}=y_{1}^{t}, \gamma_{1}=u_{1}, \ldots, \gamma_{t}=u_{t}\right\}= \\
=\max _{u_{1}, \ldots, u_{t-2 r-2} \in V} \log \mathbf{P}_{\delta}\left\{Y_{1}^{t-1}=y_{1}^{t-1}, Y_{t}=y_{t}, \gamma_{1}=u_{1}, \ldots, \gamma_{t-1}=u_{t-1}, \gamma_{t}=u_{t}\right\}= \\
=\max _{u_{1}, \ldots, u_{t-2 r-2} \in V} \log \mathbf{P}_{\delta}\left\{Y_{1}^{t-1}=y_{1}^{t-1}, \gamma_{1}=u_{1}, \ldots, \gamma_{t-1}=u_{t-1}\right\}+ \\
\quad+\log \mathbf{P}_{\delta}\left\{\gamma_{t}=u_{t} \mid \gamma_{1}=u_{1}, \ldots, \gamma_{t-1}=u_{t-1}\right\}+ \\
\quad+\log \mathbf{P}_{\delta}\left\{Y_{t}=y_{t} \mid Y_{1}^{t-1}=y_{1}^{t-1}, \gamma_{1}=u_{1}, \ldots, \gamma_{t}=u_{t}\right\} .
\end{gathered}
$$

Аналогично рассматривается случай $q>2 r+2$. Объединяя эти два случая, получаем (39). 
Следствие 1. В условиях теоремы 8 оценка $\hat{\gamma}_{1}^{T}=\left(\hat{\gamma}_{1}, \ldots, \hat{\gamma}_{T}\right)$ стегоключа по методу максимума апостериорной вероятности имеет вид

$$
\begin{aligned}
& \left(\hat{\gamma}_{T-c}, \ldots, \hat{\gamma}_{T}\right)=\arg \max _{u_{T-c}, \ldots, u_{T} \in V} \mathfrak{s}_{T}\left(u_{T-c}, \ldots, u_{T}\right), \\
& \hat{\gamma}_{t}=\arg \max _{v \in V} \mathfrak{s}_{t+c}\left(v, \hat{\gamma}_{t+1}, \ldots, \hat{\gamma}_{t+c}\right), t=T-c-1, \ldots, 1 .
\end{aligned}
$$

Доказательство. Оценка $\hat{\gamma}_{1}^{T}=\left(\hat{\gamma}_{1}, \ldots, \hat{\gamma}_{T}\right)$ стегоключа получается как обратный ход алгоритма нахождения $\max _{u_{T-c}, \ldots, u_{T} \in V} \mathfrak{s}_{T}$ согласно $(38),(39)$.

Алгоритм оценивания точек вкраплений (прямой ход (38),(39), обратный ход (40)) имеет вычислительную сложность $O\left(2^{c}+(T-c) 2^{2 c+2}\right)$.

Оценив моменты вкрапления $\gamma_{1}^{T}$ согласно (40), можно построить оценку $\hat{\xi}$ самого сообщения:

$$
\hat{\xi}_{\tau}=y_{t_{\tau}}, \quad \text { где } \quad t_{\tau}=\min _{t \in\{1, \ldots, T\}}\left\{t: \sum_{k=1}^{t} \hat{\gamma}_{k}=\tau\right\}, \tau=1, \ldots, w\left(\hat{\gamma}_{1}^{T}\right)
$$

\section{6. Результаты компьютерных экспериментов}

Приведем результаты трех серий компьютерных экспериментов на модельных данных.

Серия 1. Моделировалась исходная марковская последовательность (1) длины $T=10^{4}$ с параметром $\varepsilon=0.13$. Для $q=r=1$ согласно (3) при различных значениях параметра $\delta \in[0,1]$ моделировалась ключевая бернуллиевская последовательность и согласно (4) строилась стегопоследовательность $y_{1}^{T}$. На рис. 2 изображен график зависимости статистики числа серий $B_{T}$ от доли вкраплений $\delta$. Кружками отмечены значения статистики $\frac{1}{T-1} B_{T}$ для построенной последовательности $y_{1}^{T}$ с соответствующей долей вкраплений $\delta$, сплошной линией - график для среднего значения $\frac{1}{T-1} \mathbf{E}_{\delta}\left\{B_{T}\right\}$.

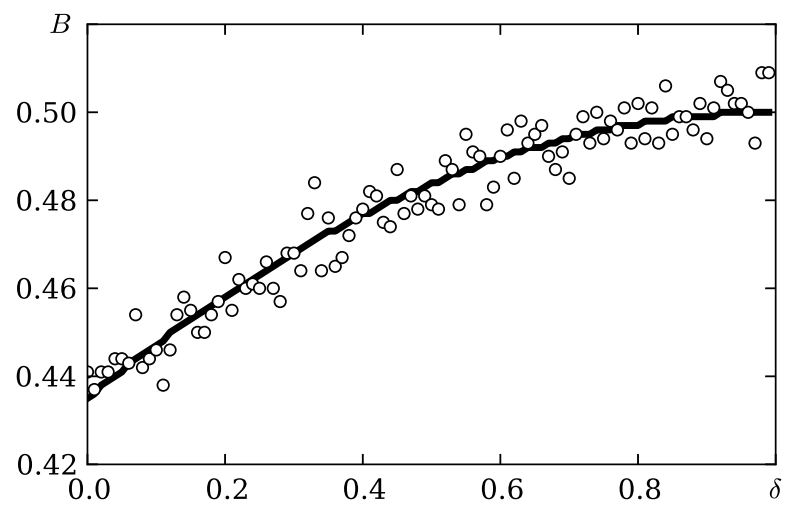

Рис. 2. Зависимость статистики числа серий $B_{T}$ от доли вкраплений $\delta$ 
Серия 2. Как и в серии 1 , методом Монте-Карло при числе прогонов $M_{1}=2^{8}$ построены оценки мощностей критериев (7), (23) проверки гипотез $H_{0, \varepsilon}, H_{1}$ при известном параметре контейнера $\varepsilon=0.48$, длине $T=2^{13}$, уровне значимости $\alpha=$ 0.05 , доле вкраплений $\delta \in\{0.005,0.01,0.015,0.02,0.025,0.03,0.04,0.05,0.06,0.07\}$.

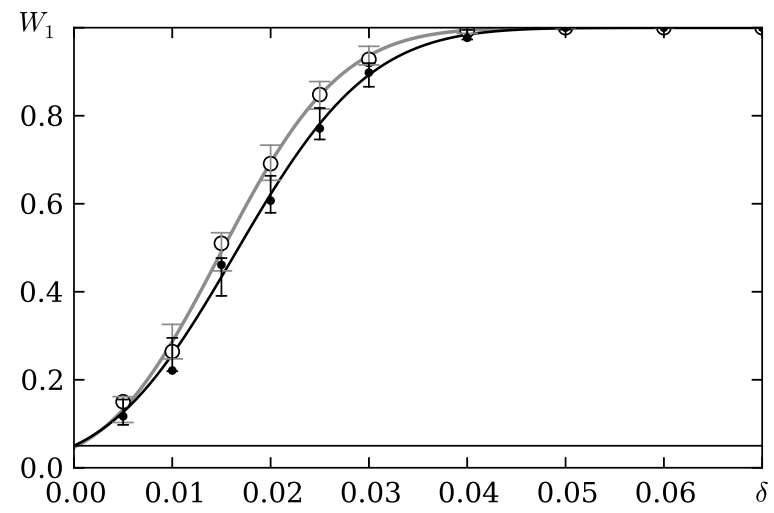

Рис. 3. Зависимость мощностей критериев $\mathscr{X}_{1 \alpha}^{B+}, \mathscr{X}_{1 \alpha}^{\mathfrak{h}+}$ от доли вкраплений $\delta$

На рис. 3 представлены графики зависимостей мощностей статистических критериев $(7),(23)$ от доли вкраплений $\delta$. Сплошной черной линией обозначена теоретическая кривая мощности критерия (7) на основе статистики числа серий, сплошной серой линией - теоретическая кривая мощности критерия (23) на основе проекции статистики коротких серий, черными кружками - оценки мощностей критерия (7), белыми кружками - оценки мощностей критерия (23). На рисунке также изображены черным и серым цветами 95\%-доверительные интервалы для оценок мощностей критериев (7) и (23) соответственно.

На графике видно, что критерий (23) на основе статистики коротких серий мощнее, чем критерий (7) на основе статистики числа серий. Численные эксперименты показывают, что при малых значениях $\varepsilon$ мощности критериев (7) и (23) практически одинаковы.

Серия 3. Для блочной модели вкраплений при $q=2, r=1$ методом Монте-Карло вычислены оценки порога $\hat{\lambda}_{\alpha}$ согласно (31) и мощности статистического критерия (29) на основе отношения правдоподобия при значениях параметров модели $\varepsilon=0.12$, длине $T=2^{18}$, уровне значимости $\alpha=0.05$. Оценка порога вычислена при числе прогонов $M_{0}=500$, оценки мощностей вычислены при числе прогонов $M_{\lambda}=250,100,200,150,100$ и доле фактического вкрапления $\delta r / q=\delta / 2$ равной $0.10,0.15,0.20,0.25,0.30$ соответственно. На рис. 4 представлен график зависимости оценок мощности критерия $\mathscr{X}_{1 \alpha}^{\lambda}$ от доли фактического вкрапления $\delta / 2$.

Результаты компьютерных экспериментов иллюстрируют работоспособность построенных статистических критериев обнаружения вкраплений, а также согласие численных результатов с теоретическими.

В заключение отметим, что для обнаружения вкраплений могут использоваться малопараметрические модели цепей Маркова высокого порядка [15]. 


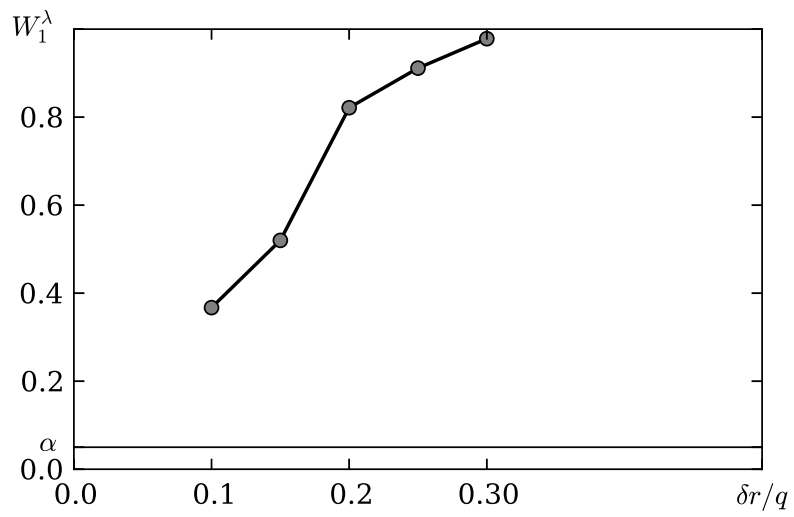

Рис. 4. Зависимость мощности критерия $\mathscr{X}_{1 \alpha}^{\lambda}$ от доли вкраплений $\delta r / q$

Авторы благодарны А.М. Зубкову за советы по исследованию статистик серий для обнаружения вкраплений и рецензентам за замечания и рекомендации.

\section{Список литературы}

1. Пономарев К. И., "Параметрическая модель вкрапления и ее статистический анализ", Дискретная математика, 214:4 (2009), 148-157.

2. Пономарев К. И., "Об одной статистической модели стеганографии", Дискретная математика, 21:2 (2009), 138-145.

3. Ker A., "A capacity result for batch steganography", IEEE Signal Process. Lett., 14:8 (2007), $525-528$.

4. Шойтов А.М., "О выявлении факта зашумления конечной цепи Маркова с неизвестной матрицей переходных вероятностей", Прикладная дискретная математика, Приложение № $3,2010,44-45$.

5. Харин Ю. С., Вечерко Е. В., "Статистическое оценивание параметров модели вкраплений в двоичную цепь Маркова", Дискретная математика, 25:2 (2013), 135-148.

6. Зубков А. М., “Датчики псевдослучайных чисел и их применения", Труды II Междунар. научн. конф. "Математика и безопасность информационных технологий", 2003, 200-206.

7. Кемени Дж. Г., Снелл Дж. Л., Конечные цепи Маркова, Наука, Москва, 1970.

8. Иванов В.А., “Модели вкраплений в однородные случайные последовательности”, Труды по дискретной математике, 10 (2008), 18-34.

9. A statistical test suite for random and pseudorandom number generators for cryptographic applicatiions: NIST Special Publication 800-22 Rev. 1a., Nat. Inst. Stand. Technol., 2010.

10. Doukhan P., Mixing: properties and examples, Springer-Verlag, 1994.

11. Kharin Yu. S., Voloshko V. A., "Robust estimation of AR coefficients under simultaneously influencing outliers and missing values", J. Statist. Plan. Infer., 141:9 (2011), 3276-3288.

12. Ивченко Г. И., Медведев Ю. И., Математическая статистика, Высшая школа, Москва, 1984.

13. Wald A., "Tests of statistical hypotheses concerning several parameters when the number of observations is large", Trans. Amer. Math. Soc., 54:3 (1943), 426-482. 
14. Rabiner L. R., "A tutorial on hidden Markov models and selected applications in speech recognition", Proc. IEEE, 77:2 (1989), 257-286.

15. Ю. С. Харин, А. И. Петлицкий, "Цепь Маркова $s$-го порядка с $r$ частичными связями и статистические выводы о ее параметрах", Дискретная математика, 19:2 (2007), 109-130.

Статья поступила 31.03.2015. 